\title{
Médiévales
}

Langues, Textes, Histoire

61 | automne 2011

La chair des émotions

\section{La narration des émotions et la réactivité du destinataire dans Les Contes de Canterbury de Geoffrey Chaucer}

The Narration of Emotions and the Reader's Reactions in Geoffrey Chaucer's

Canterbury Tales

\section{Guillemette Bolens}

\section{OpenEdition}

Journals

Édition électronique

URL : https://journals.openedition.org/medievales/6298

DOI : $10.4000 /$ medievales. 6298

ISSN : $1777-5892$

\section{Éditeur}

Presses universitaires de Vincennes

\section{Édition imprimée}

Date de publication : 20 décembre 2011

Pagination : $97-117$

ISBN : 978-2-84292-337-2

ISSN : 0751-2708

\section{Référence électronique}

Guillemette Bolens, «La narration des émotions et la réactivité du destinataire dans Les Contes de Canterbury de Geoffrey Chaucer », Médiévales [En ligne], 61 | automne 2011, mis en ligne le 19 janvier 2012, consulté le 22 avril 2022. URL : http://journals.openedition.org/medievales/6298 ; DOl : https:// doi.org/10.4000/medievales.6298 
Médiévales 61, automne 2011,p.97-118

\title{
Guillemette BOLENS
}

\section{LA NARRATION DES ÉMOTIONS ET LA RÉACTIVITÉ DU DESTINATAIRE DANS LES CONTES DE CANTERBURY DE G. CHAUCER}

\author{
Lo, which a greet thyng is affeccioun! \\ Men may dyen of ymaginacioun, \\ So depe may impressioun be take. \\ This sely carpenter bigynneth quake. \\ The Canterbury Tales, «The Miller's Tale», 3611-3614. \\ [Ah, quelle grande chose est l'émotion! \\ Les hommes peuvent mourir de leur imagination, \\ Tant est profonde l'impression que l'on peut en tirer. \\ Ce simple charpentier commença à trembler.]
}

Écrite à la fin du XIV e siècle, l'œuvre de Geoffrey Chaucer Les Contes de Canterbury est un texte littéraire qui montre que leur auteur avait une perception fine des enjeux engagés dans les constructions sociales des phénomènes cognitifs et corporels associés à ce que nous appelons aujourd'hui émotions. Cette œuvre majeure met en évidence à tous les niveaux du récit les rapports de force complexes qui s'actualisent à travers l'expression verbale et corporelle des émotions. Ces rapports de force sont manifestes entre les personnages des Contes de Canterbury, mais ils impliquent également les destinataires du texte. L'écrivain Chaucer, quand il met en récit l'expression corporelle d'une émotion, lance un défi interprétatif à ses destinataires. Mon objectif est ainsi de chercher à comprendre ce que le texte signale de nos modalités d'accès à un sens émotionnel permis par une narration des signes corporels. Plutôt que de localiser des motifs émotionnels dans le texte littéraire, il va s'agir de comprendre comment le texte met le corps en récit, afin d'augmenter nos chances de saisir les signes corporels qui l'habitent, évitant ainsi de réduire ceux-ci à des équations simplificatrices du type tel geste (par exemple un sourire) signifie tel concept (par exemple la joie) ${ }^{1}$.

1. Sur l'expression faciale comme geste, voir K. THомAs dans son introduction à A Cultural History of Gesture: "gesture includes any kind of bodily movement or posture (including facial expression) which transmits a message to the observer », dans J. BREMMER et H. Roodenburg éd., A Cultural History of Gesture, Ithaca, 1992, p. 1-14 (ici p. 1). 
Dans ce but, le style kinésique est une notion utile, qui renvoie à la facture particulière des gestes et des mouvements ${ }^{2}$. En situation réelle, le style kinésique concerne la manière de bouger d'une personne, à l'inclusion des nuances les plus ténues. Tandis que le style kinésique du corps réel se manifeste dans toutes les nuances expressives des mouvements, le style kinésique d'une œuvre littéraire se perçoit dans la dynamique discursive et narrative qui donne lieu à la compréhension d'événements corporels stylisés par la langue. Dans un texte, l'analyse kinésique consiste à distinguer aussi précisément que possible, par la facture du récit, la dynamique de l'espace narratif créée par l'expression gestuelle et motrice, telle qu'elle a été traduite langagièrement. L'analyse kinésique en littérature consiste à observer les moyens exacts choisis dans l'œuvre pour signifier par la référence au mouvement corporel.

Dans le cas de Chaucer, ses œuvres n'ont de cesse de piéger leur destinataire en créant des attentes qui poussent le lecteur inattentif au dérapage interprétatif, entre autres concernant la manifestation corporelle des émotions. Certains passages signalent avec force que l'auteur met délibérément à l'épreuve l'agilité interactionnelle de son destinataire. Pour entrer dans cette joute épistémologique, il faut très bien connaître les intertextes de l'œuvre ainsi que les codes gestuels pertinents dans la période et la culture de l'auteur. Mais cela ne suffit jamais. Il faut également utiliser cognitivement son propre savoir sensori-moteur afin d'attraper le texte au vol. Ainsi, mon objectif est de tenir compte des techniques narratives de l'œuvre en vue de traiter d'une expression corporelle dans «Le Conte de l'Écuyer», celle de l'évanouissement par frustration amoureuse - chez un oiseau. Par cette gageure, je chercherai à relever le défi lancé par Geoffrey Chaucer il y a six siècles, à travers un acte de lecture qui prend l'humour complexe de cet écrivain médiéval suffisamment au sérieux pour entrer dans son jeu.

Chaucer brouille régulièrement la piste de son destinataire. Il le perd dans un réseau d'informations souvent contradictoires. Par ce jeu narratologique, Chaucer confronte son lecteur au processus sémantique par lequel celui-ci élabore un sens à partir du texte. John Ganim met en évidence cet aspect de la poétique chaucérienne qui consiste à promouvoir la més-interprétation (misinterpretation), l'interprétation manquée ou erronée ${ }^{3}$. Quant à R. W. Hanning, il met l'accent sur la manière qu'a Chaucer de pratiquer ce qu'il appelle le «harcèlement textuel». Par cette expression, Hanning désigne cet aspect récurrent chez Chaucer qui consiste à introduire des erreurs dans les traductions de textes bien connus,

2. J'élabore la question du style kinésique dans l'introduction du Style des gestes. Corporéité et kinésie dans le récit littéraire, Lausanne, 2008, p. 17-24; traduction anglaise à paraître chez Johns Hopkins University Press.

3. J. M. GANIM, Chaucerian Theatricality, Princeton, 1990, p. 44. 
à citer à faux ou hors contexte, et à abuser des autorités textuelles ${ }^{4}$. C'est ce que nous observerons, en un second temps, par une analyse de «L'Introduction et Prologue du Juriste». L'expression émotionnelle du corps sera alors à observer dans l'interface entre le texte et son destinataire, seul espace auquel nous avons effectivement accès dans notre effort de compréhension des émotions médiévales.

Le récit cadre des Contes de Canterbury montre une trentaine de pèlerins partant d'une taverne dans la banlieue de Londres pour se rendre en pèlerinage à Canterbury, où les reliques de saint Thomas Becket sont vénérées. Parmi les pèlerins se trouve un narrateur qui porte le nom de l'auteur, soit Chaucer. Le tavernier qui héberge les pèlerins décide au matin de les accompagner, et propose un jeu pour rendre la route plus agréable: chaque pèlerin devra raconter une ou deux histoires à l'aller et au retour, et le meilleur récit sera récompensé par un souper offert. En définitive, le verdict ne sera jamais prononcé, car le texte s'achève par une rétraction du narrateur avant que les pèlerins n'arrivent à Canterbury. Cette rétraction clôt, en faisant mine de la désavouer, une série de contes, ainsi que des prologues, des épilogues et des liens narratifs entre les contes, pendant lesquels les pèlerins se parlent et souvent se disputent. Pour cerner la question du style kinésique de cette poétique, je vais me concentrer sur deux sections des Contes de Canterbury, à savoir «Le Conte de l'Écuyer» et «L'Introduction et le Prologue de l'homme de loi».

Le style kinésique de la poétique de Chaucer correspond à un art du lapsus feint. Par lapsus j'entends l'acception courante de dérapage verbal symptomatique d'un sens réprimé. Or, je l'associe paradoxalement à l'idée de maîtrise et de feinte délibérée, car l'analyse qui va suivre tend à montrer que les dérapages textuels, si caractéristiques de la poétique chaucérienne, sont le résultat d'un art consommé. Cet art met le lecteur au défi de se repositionner constamment, et le style kinésique de cette poétique provoque des réactions indissociablement cognitives, émotionnelles et corporelles, dont la plus évidente est celle du rire.

L'héroïne principale du «Conte de l'Écuyer» («The Squire's Tale») est une princesse du nom de Canacé 5 . Ce nom nous conduit en ligne intertextuelle

4. R.W. Hanning, «Roasting a Friar, Mis-taking a Wife, and Other Acts of Textual Harassment in Chaucer's Canterbury Tales», Studies in the Age of Chaucer, 7 (1985), p. $3:$ «One of the most characteristic, and remarkable, features of Chaucer's Canterbury Tales is its penchant for what can perhaps best be called textual harassment. The Canterbury pilgrims - and indeed the characters within their tales - harass texts almost as frequently, and enthusiastically, as they harass each other. That is, they misquote, quote out of context, misinterpret, vulgarize, and generally abuse textual "auctoritee".»

5. Sur le «Squire's Tale», voir entre autres A. S. Ambrisco, «"It Lyth Nat in My Tonge": Occupatio and Otherness in the "Squire's Tale"», The Chaucer Review, 38/3 (2004), p. 205-228; W. Kamowski, «Trading the "Knotte" for Loose Ends: The "Squire's Tale" and the Poetics of Chaucerian Fragments », Style, 31/3 (1997), p. 391-412; L. M. JonES, «Chaucer's Anxiety of Poetic 
directe aux Héroïdes d'Ovide, où Canacé est la protagoniste épistolaire d'un drame atroce d'inceste et d'infanticide. Or, à la place d'un récit de transgression incestueuse et de meurtre sur nouveau-né - un récit bien connu à l'époque et mentionné ailleurs dans les Contes de Canterbury -, nous avons affaire à un échange entre un faucon femelle et Canacé, dont la bague magique lui permet de comprendre les oiseaux. L'échange se fait en deux parties. Dans la première partie, Canacé entend les plaintes de l'oiseau qui se frappe de son bec au point de s'évanouir de façon répétée pour finalement tomber de son arbre; la deuxième partie de l'échange est un dialogue où Canacé s'enquiert des raisons de tant de douleur et où l'oiselle explique qu'elle souffre d'avoir été trahie par son amant, un faucon parti avec une autre.

L'événement corporel émotionnel qui m'intéresse est la chute de l'oiseau qui s'évanouit de douleur amoureuse. Par définition capable de voler, l'oiseau, dans «Le Conte de l'Écuyer», choit. Or, cette expression émotionnelle physique a lieu devant la princesse Canacé qui, malgré son empressement très courtois, tend ses jupes mais manque l'animal, lequel s'écrase au sol. Étonnamment, l'humour de cet événement a longtemps été oblitéré. Car l'évanouissement est une expression émotionnelle qui n'est pas rare dans la littérature médiévale pour exprimer l'intensité insoutenable d'un sentiment, que ce sentiment soit amoureux ou non - par exemple dans le Conte du Graal de Chrétien de Troyes, Arthur s'évanouit d'inquiétude pour son neveu Gauvain (9220-9223 : «Et chiet pasmez de la destrece», il choit pâmé de détresse parce qu'il ne le voit pas parmi les autres chevaliers $)^{6}$. Cette expression émotionnelle est fortement développée dans une autre œuvre de Chaucer, Trö̈lus and Crisseyde, où l'amant s'évanouit au moment où il se retrouve enfin au bord du lit de celle qu'il aime (livre III, 1. 1092: «And down he fel al sodeynly aswowne» [«et il tomba tout soudainement évanoui»]). Fréquent dans de nombreux textes moyen anglais, l'évanouissement, du vieil anglais gesogung, geswowung, qui donnera l'anglais moderne swoon, apparaît dans quatre autres contes des Canterbury Tales («The Knight's Tale», «The Clerk's Tale», «The Franklin's Tale» et «The Physician's Tale»). Il est donc possible que le destinataire érudit, reconnaissant une expression émotionnelle «normale», n'en saisisse pas l'incongruité, quand l'événement narré se nuance

Craft: The "Squire's Tale"», Style, 41/3 (2007), p. 300-382 ; S. SHARon-ZisSER, «The "Squire's Tale" and the Limits of Non-Mimetic Fiction», Chaucer Review, 26/4 (1992), p. 377-394; C. A. BERRY, «Flying Sources: Classical Authority in Chaucer's "Squire's Tale” , ELH, 68 (2001), p. 287-313; E. SCALA, «Canacee and the Chaucer Canon: Incest and Other Unnarratables», Chaucer Review, 30/1 (1995), p. 15-39. Scala offre une analyse psychanalytique de la légende de Canacé chez Chaucer, en mettant l'accent sur «l'anxiété critique» (critical anxiety) générée par l'association de Canacé à un drame incestueux.

6. Voir P. Levron, «Mélancolie, émotion et vocabulaire», dans D. Boquet et P. NAgy éd., Le Sujet des émotions au Moyen Âge, Paris, 2009, p. 258. 
de jupes tendues au mauvais endroit. Ma proposition méthodologique sera donc d'augmenter l'attention que nous portons aux visualisations que nous générons à la lecture d'un texte, en particulier quand ces visualisations concernent des mouvements corporels ${ }^{7}$.

\section{Canacé et les risques de la lecture}

La légende de Canacé offre à Chaucer, mais aussi à John Gower, auteur anglais contemporain de Chaucer, l'occasion de provoquer leurs destinataires. Pour saisir le contraste entre les deux traitements de la légende et l'originalité de Chaucer, commençons par Gower. Le cadre narratif de la Confessio amantis de Gower met en scène un amant en désarroi, qui progressivement apprend la nature des péchés capitaux ${ }^{8}$. L'histoire de Canacé apparaît dans le chapitre qui concerne le péché de colère, en anglais wrath. Cependant, l'inceste entre Canacé et son frère Macarée n'est étrangement pas la raison pour laquelle leur triste aventure est offerte en guise d'exemplum; le frère et la sœur ne sont pas coupables du péché capital illustré par cette section de l'œuvre. Ce sont la colère et la cruauté incontrôlées de leur père que l'exemplum dénonce, comme ce père, après avoir compris la relation incestueuse de ses enfants, oblige sa fille Canacé à se suicider, et jette le fils dont elle vient d'accoucher en pâture aux bêtes sauvages. Quant à Macarée, il s'est déjà enfui sans se retourner. Par cette version des faits, Gower est à la fois fidèle au récit tel qu'Ovide le raconte dans ses Hérö̈des, et en rupture avec l'interprétation que laisse prévoir l'utilisation de ce récit dans un discours sur les péchés. Ainsi, Gower joue avec les attentes du lecteur mais d'une façon qui diffère de celle de Chaucer, comme elle concerne la problématique du jugement éthique ${ }^{9}$.

Dans les versions d'Ovide et de Gower, le père joue un rôle essentiel. D'une cruauté monstrueuse, il fait porter un glaive à Canacé qui, obéissante, se trucide. Il vient ensuite saisir l'enfant baignant dans le sang maternel et, insensible aux vagissements du nouveau-né, part au plus profond de la forêt jeter son petitfils aux bêtes, là où il est sûr que nul ne pourra le secourir. L'extrême violence de cette légende marque définitivement le nom de Canacé. D'une part, ce nom n'est jamais réinvesti autrement dans la littérature occidentale; d'autre part, le texte des Contes de Canterbury fait dire à l'un de ses personnages (nous y viendrons) que Chaucer ne se commettrait jamais à raconter la légende de Canacé, soit ce

7. Je traite de la question des simulations perceptives (par ex. les visualisations de mouvements) dans Le Style des gestes, op. cit.

8. John Gower, Confessio amantis, éd. R. A. PeCK, Toronto-Londres, 1980.

9. Voir S. Echard éd., A Companion to Gower, Woodbridge, Suffolk, 2004. 
récit d'infanticides en série. Dès lors, quand ce nom apparaît ailleurs dans la même œuvre, il est fortement associé aux idées de crime familial et d'inceste.

Cette association alimente une théorie du suspens, énoncée par l'Écuyer au moment de focaliser son récit sur sa Canacé. L'Écuyer explique qu'un conteur ne doit pas retarder le «nœud» (knot) de son récit, car il risque alors de perdre l'attention de son auditoire.

The knotte why that every tale is toold, If it be taried til that lust be coold Of hem that han it after herkned yoore, The savour passeth ever lenger the moore, For fulsomnesse of his prolixitee.
Le nœud qui fait l'intérêt d'une histoire, $\mathrm{Si}$ on met trop de temps à le nouer Au point de refroidir son auditoire, Perd du goût à mesure que le temps passe : La prolixité génère l'ennui ${ }^{10}$.

Le suspens est théorisé par le narrateur du conte, induisant, précisément, du suspens: on s'attend au pire. Or, surprise, les gestes fatals ne sont jamais perpétrés, le nœud ne se noue jamais, et le destinataire reste empêtré dans ses hypothèses interprétatives ${ }^{11}$. Tandis que le nom de l'héroïne pousse à prévoir un drame, la narration retarde ce point nodal jusqu'à le supprimer entièrement. La théorie de l'Écuyer ouvre sur un récit qui piège le lecteur et le pousse au dérapage, pour cette raison que le texte efface précisément les traces d'inceste. En effet, à la place de la transgression incestueuse et du meurtre intolérable, nous avons affaire à un échange doucereux entre un faucon femelle et une Canacé aux émotions conventionnelles. Quand la fauconnette explique plus loin qu'elle souffre d'avoir été trahie par son amant à plumes, le volatile reproduit tous les signes véhéments d'un amour courtois hyperbolique. Et avant même de connaître la nature du drame personnel de l'oiselle, Canacé a un comportement qui représente avec humour le style kinésique de la poétique chaucérienne, telle qu'elle s'incarne à travers le conteur aux dons limités qu'est l'Écuyer.

En effet, le volatile se frappe de ses ailes et de son bec au point de se faire saigner abondamment. Il s'évanouit de façon répétée à force de perdre du sang, et la jeune fille, l'apercevant, se précipite vers l'arbre sur lequel il se tient.

And wel neigh for the routhe almoost she dyede

And to the tree she gooth ful hastily,

And on this faukon looketh pitously,

And heeld hir lappe abrood, for wel
Et, saisie, de pitié, [Canacé] en mourut presque

Et en toute hâte elle s'approche de l'arbre

Et elle regarde l'oiselle avec compassion.

Et elle tendit un pan de sa robe, sachant bien

10. Geoffrey Chaucer, The Canterbury Tales dans The Riverside Chaucer, éd. L. D. Benson, $3^{\text {e }}$ éd., Oxford, Oxford University Press, 1987, «The Squire's Tale», 1. 401-405. Traduction d'A. CrépIn, Les Contes de Canterbury, Paris, 2000.

11. Cet inconfort a souvent été résolu en mettant fortement en doute les compétences narratives et rhétoriques du conteur, en l'occurrence l'Écuyer. Les références de la note 5 traitent de cet aspect de l'histoire critique du «Conte de l'Écuyer». 
she wiste

The faukon moste fallen fro the twiste, Whan that it swowned next, for lak of blood.

A longe whil to wayten hire she stood [...]

Tho shrighte this faucon yet moore pitously

Thane ever she dide, and fil to grounde anon,

And lith aswowne, deed and lyk a stoon, Til Canacee hath in hire lappe hire take Unto the tyme she gan of swough awake.
Que la fauconnette perdrait l'équilibre Quand, faute de sang, elle s'évanouirait.

Elle la guetta ainsi un long temps

[...]

Alors l'oiselle poussa un cri plus lamentable

Que tous les autres, et elle tomba par terre,

Évanouie, sans vie, telle une pierre,

Canacé la mit au creux de sa robe

Jusqu'à ce qu'elle commence à reprendre connaissance $^{12}$.

Risquer mourir de pitié en entendant les cris d'un faucon opère d'emblée comme une déflation du sérieux supposé de la narration, et ce quand bien même Canacé comprend le langage des oiseaux. Une sensibilité exagérée à un pathos encore immotivé produit un effet inverse. Le lecteur n'a pas les moyens de partager la sympathie incandescente de l'hérö̈ne puisque ni celle-ci ni le narrateur ne connaissent les raisons de tant d'émotions. La princesse fera bientôt passer à l'oiseau un interrogatoire en règle, faisant des hypothèses empreintes de conventionnalisme avant de se taire enfin pour laisser la fauconnette s'expliquer. Ensuite, la jeune fille tend les pans de sa robe car elle anticipe une chute au prochain évanouissement, restant ainsi un long temps, toute robe tendue. Pourtant, lorsque le volatile finit par choir, elle le manque, et il reste comme mort sur le sol, jusqu'à ce que la jeune fille le ramasse et le dépose sur ses jupes.

Le système de référence de cette partie du conte est clairement aristocratique et courtois: la fauconnette utilise les topoi de la fin'amor dans sa grande plainte et le récit de ses amours déçues. Cela a fait dire à Ian Robinson que «The Squire's Tale» est «the only Canterbury tale about pure courtly love» ${ }^{13}$. Voir dans ce texte de l'amour courtois pur suggère que la chute de l'oiseau est prise sérieusement. Ou plutôt, la chute est oblitérée, comme c'est le cas dans l'interprétation proposée par Lesley Kordecki, qui n'en fait pas mention dans son analyse de cette partie du conte et pour qui «we respond to the purity of lament» ${ }^{14}$. Comme l'a souligné John McCall, le plus étonnant au sujet de ce

12. Geoffrey Chaucer, The Canterbury Tales, op. cit., «The Squire's Tale», 1. 438-444; 472476 (trad. A. CRÉPIN). Je me permets d'apporter quelques modifications à l'excellente traduction d'André Crépin dans le but de la rendre plus littérale, au risque de perdre son élégance, mais afin de soutenir mon argument.

13. I. Robinson, Chaucer and the English Tradition, Cambridge, 1972, p. 181-182.

14. L. KordeCKI, «Chaucer's "Squire's Tale": Animal Discourse, Women, and Subjectivity», The Chaucer Review, 36. 3, 2002, p. 289. 
conte est que son humour a pu - et manifestement peut toujours - être évacué ${ }^{15}$. La question n'est pas d'étiqueter ce conte comme parodique. Ce serait simplifier la poétique de Chaucer que de la réduire à une série de genres. Sans vouloir nier l'intérêt des autres aspects de ce conte, il me paraît important de mettre l'accent sur un élément kinésique souvent omis par la critique. Car la chute de l'oiseau évoque la poétique chaucerienne, laquelle met son lecteur au défi de tomber dans ses pièges - ou à côté de ses jupes. La chute de la fauconnette incarne un style kinésique où le texte se fait tomber comme un oiseau hyperémotif; il fait des lapsus délibérés, lesquels fonctionnent comme autant de dérapages soumis à la réactivité du lecteur et à ses réflexes interprétatifs.

Premièrement, le nom même de Canacé alourdit la réception du récit: le lecteur s'attend continuellement au pire (inceste, infanticide, etc.). Il est préparé à lire avec sérieux un drame abominable. Cette gravité empreint sa réception du récit de façon globale. Or la gravité, par définition, alourdit. À l'inverse du lapsus verbal qui fait dire malgré toute attente ce qui devait être tu, le texte ne dit pas les crimes attendus. Et c'est cela précisément qui suspend l'agilité du destinataire, lequel manque l'humour d'une chute. Le lapsus littéral de l'oiseau (sa chute) joue le piège tendu au lecteur qui, comme Canacé éplorée sans vraiment savoir pourquoi, tend ses jupes au mauvais endroit et rate son objet. Car le nœud du suspense dans ce conte est l'absence du nœud attendu : ni inceste ni infanticide. À ce jeu, Chaucer éprouve la mobilité de son lecteur.

On ne peut lire un amour idéalisé et idéalisable dans «The Squire's Tale» qu'à la condition d'effacer la chute de l'oiseau et la facture particulière de cette chute. Et c'est donc par un travail attentif de visualisation kinésique que le lecteur peut éviter de passer à côté de l'œuvre. La visualisation kinésique ici doit impérativement tenir compte du fait que le texte nous montre Canacé tenant ses robes tendues pour attraper l'oiseau, attendant un long temps, pour en définitive voir l'animal tomber à côté. La simulation produit l'inférence kinésique significative d'une chute à côté. Tout est là. Si le lecteur ne produit pas cette inférence kinésique, c'est à côté de Chaucer qu'il passe.

Une deuxième raison permet d'expliquer l'absence de réaction critique devant la chute de la fauconnette. Les oiseaux sont souvent chargés d'incarner l'expression du désir ${ }^{16}$, que ce désir concorde avec le renouveau printanier ou qu'il s'associe à une souffrance de séparation (par exemple chez Marie de France dans le lai «L'Aüstic»), à de la jalousie (chez Bernard de Ventadour ${ }^{17}$ ) voire à

15. J. MCCALL, « The Squire in Wonderland », Chaucer Review, 1/2 (1966), p. 103-109.

16. Sur l'importance de l'association des oiseaux et du désir, voir M. ZiNK, Nature et poésie au Moyen Âge, Paris, 2006.

17. Bernard de Ventadour, Chansons d'amour, éd. et trad. M. Lazar, Paris, 1966, p. 180$181, \mathrm{n}^{\circ} 31$ : «Can vei la lauzeta mover/de joi sas alas contral rai,/que s'oblid'e. s laissa chazer/per la 
l'ascension spirituelle et au plaisir éternel, chez Dante, qui place l'alouette de Ventadour au Paradis, là où l'oiseau suspendu dans son vol devient «l'image de l'empreinte du plaisir éternel (l'imago de la 'mprenta de l'etterno piacere) ${ }^{18}$ ». Face à la puissance de ces intertextes, l'oiseau de Chaucer tombe plus fort encore, et avec lui les idéaux de fin'amor et de sublime divin. On ne peut lire une grande pureté dans «The Squire's Tale» qu'à la condition de camoufler la chute de l'oiseau. Car cet oiseau-là, au lieu d'être en suspension dans un désir parfait qui le porte, s'écrase au sol.

Troisièmement, comme je l'ai déjà souligné, l'évanouissement n'est pas rare dans l'expression émotionnelle médiévale, et l'habitude peut émousser la réactivité interprétative. L'amoureux s'évanouit: rien à signaler, signe normal d'amour courtois. Or, la scène de l'oiseau est chez Chaucer précisément un commentaire sur l'intégration culturelle des systèmes conceptuels. Dans «Le Conte de l'Écuyer», l'utilisation du système de l'amour courtois sert à signifier que ce système informe les comportements et les anticipations motrices. J'utilise le verbe informer dans ses deux acceptions de donner une forme et de fournir une information permettant de comprendre les signes kinésiques. Dit autrement, à la fin du XIve siècle, un amoureux ou une amoureuse, ça risque de s'évanouir, et on s'y attend au point de tendre ses jupes pour le ou la rattraper. Reste qu'ici elle est un oiseau qui s'évanouit à force de s'automutiler et qu'elle tombe à côté.

Cela fait penser que l'espace narratif créé par l'œuvre au moment de la réception nécessite une alliance entre, d'une part, un savoir historique, culturel et intertextuel et, d'autre part, une intelligence kinésique, c'est-à-dire la faculté de sémantiser et de comprendre les mouvements corporels, les postures, les gestes et les expressions faciales ${ }^{19}$. En un mot, le destinataire des feintes de l'auteur Chaucer ne doit pas seulement connaître ses intertextes et savoir que les émotifs médiévaux ont en littérature une certaine tendance à s'évanouir, il doit pondérer

doussor c'al cor li vai,/ai ! tan grans enveya m'en ve/de cui qu'eu veya jauzion,/meravilhas ai, car desse/lo cor de dezirer no. m fon.» [ «Quand je vois l'alouette mouvoir de joie ses ailes contre les rayons du soleil, perdre conscience et se laisser choir à cause de la douceur qui pénètre son cœur, hélas ! une si grande jalousie me vient de tous ceux que je vois réjouis : je suis étonné que mon cœur aussitôt ne s'évanouisse de désir!»]

18. Dante Alighieri, La Divine Comédie : Le Paradis, canto XX, 73-78, éd. et trad. J. RisSEt, Paris, Flammarion, 1992 (1990): «Quale allodetta che 'n aere si spazia/prima cantando, et poi tace contenta/de l'ultima dolcezza che la sazia,/tal mi sembiò l'imago de la 'mprenta/de l'etterno piacere, al cui disio/ciascuna cosa qual ell'è diventa.» [«Comme l'alouette qui s'élance dans l'air chantant d'abord, et puis se tait, contente de la dernière douceur qui la comble, telle me sembla l'image de l'empreinte du plaisir éternel, au désir de qui toute chose devient ce qu'elle est.»]

19. Sur l'intelligence kinésique, voir E. Spolsky, «Elaborated Knowledge: Reading Kinesis in Pictures », Poetics Today, 17/2 (1996), p. 157-180; G. Bolens, Le Style des gestes, op. cit. 
son recours aux typologies socioculturelles des gestes, pour se permettre de réagir cognitivement au récit d'une chute, voire d'en rire ou d'en pleurer.

\section{Le personnage du Juriste face à l'auteur Chaucer}

Chez Chaucer, l'intertextualité joue un rôle majeur en ce sens que la lecture du texte nécessite une connaissance d'autres textes pour que les jeux sémantiques de l'auteur puissent être saisis. «L'Introduction et le Prologue de l'Homme de loi » thématise cette question très précisément, d'autant plus que les intertextes employés sont aussi bien les œuvres de divers auteurs connus que celles de Chaucer lui-même. Et s'il faut les connaître, c'est pour se rendre compte que Chaucer introduit des travers dans sa manière de faire référence à ses propres textes aussi bien qu'à ses sources extérieures. Par l'analyse qui suit, je cherche à mettre en évidence la place donnée dans la poétique chaucérienne à la réactivité du destinataire. Ceci nous conduira au personnage du Juriste, qui affirme que Chaucer ne se commettrait jamais à écrire sur Canacé.

Commençons avec l'intertexte d'un auteur bien connu à l'époque et reconnu comme auctor, soit comme une source d'autorité culturelle et religieuse. Il s'agit du pape Innocent III. Le prologue du Juriste contient une traduction d'une section du De miseria condicionis humane d'Innocent III, un texte portant sur la misère de la condition humaine. Innocent III (Giovanni Lotario, comte de Segni) fut pape de 1198 à 1216; il est considéré comme le plus puissant pape du Moyen Âge. La section de son De miseria repris par Chaucer s'appelle De miseria pauperis et divitis [ «De la misère des pauvres et des riches»]. Le pape y dénonce la vanité des fausses richesses, montrant que les richesses temporelles sont une autre forme de misère. Chaucer met dans la bouche de son Juriste en pèlerinage une traduction fidèle de la portion du texte qui décrit la cruauté de l'indigence, pour soudain rompre avec son modèle au moment de parler des riches, en concluant de façon parfaitement inattendue par une louange des marchands fortunés.

L'argument du pape tient en deux volets: le premier volet décrit le malheur des pauvres, et le deuxième volet dénonce la misère humaine des riches. Les trois premières strophes traduites par Chaucer sont clairement proches du texte d'Innocent III, y compris par les références bibliques ${ }^{20}$. En revanche, les deux

20. Lotario Dei Segni (Pope Innocent III), De miseria condicionis humane, I. 14; « De miseria pauperis et divitis», R. E. LewIs éd. et trad., The Chaucer Library, Athens (Georgia), 1978 : «Pauperes enim premuntur inedia, cruciantur erumpna, fame, siti, frigore, nuditate: vilescunt et contabescunt, spernuntur et confunduntur. O miserabilis condicio mendicantis! Et si petit, pudore confunduntur, et si non petit, egestate consumitur, set ut mendicet necessitate compellitur. Deum causatur iniquum quod non recte dividat; proximum criminatur malignum quod non plene subveniat; indignatur, 
dernières strophes pervertissent brusquement le discours d'origine. Dans son deuxième volet, Innocent III écrit ceci :

Quelle honte ! Une personne est estimée selon sa fortune, quand la fortune devrait être estimée à l'aune de la personne. Un tel est réputé aussi bon qu'il est riche, et aussi mauvais qu'il est pauvre, quand il devrait plutôt être réputé aussi riche qu'il est bon, et aussi pauvre qu'il est mauvais. Le riche, pourtant, est désinhibé par l'excès de biens et sans frein dans sa jactance, il se précipite vers son plaisir et s'écroule dans l'illicite, et les choses qui constituaient les amusements de ses péchés deviennent les instruments de ses peines. Labeur dans l'acquisition, crainte dans la possession, douleur dans la perte fatiguent, sollicitent et affligent toujours l'esprit du riche. «Là où se trouve ton trésor, se trouve aussi ton cœur.» ${ }^{21}$.

Le discours papal est normatif, condamnant les émotions associées à l'acquisition laborieuse et à l'appétit de richesse. Or, Chaucer exploite la normativité de sa source pour produire un décalage qui, en tant que décalage, peut avoir un impact cognitif, émotionnel et corporel sur le destinataire, par exemple par la confusion, l'approbation, le rire, le dédain, la colère, ou autre - selon les dispositions affectives, intellectuelles et idéologiques du destinataire. Pour saisir cet acte discursif chaucérien, il est important d'avoir accès au passage entier, dans lequel le retournement subversif a lieu comme par mégarde.

O hateful harm, condicion of poverte!

With thurst, with coold, with hunger so confoundid!

To asken help thee shameth in thyn herte;
Ô mal détestable, vie de pauvreté

Proie de la soif, du froid, et de la faim.

Demander secours t'emplit l'âme de honte

murmurat, inprecatur. Adverte super hoc sentenciam sapientis: "Melius est mori quam indigere." "Eciam proximo suo pauper odiosus erit." "Omnes dies pauperis mali." "Fratres hominis pauperis oderunt eum, insuper et amici procul recesserunt ab eo." "Cum fueris felix, multos numerabis amicos. Tempora si si fuerint nubila, solus eris.” " [ «The poor are indeed oppressed by starvation, tortured by need, hunger, thirst, cold, nakedness; they become worthless and waste away, are despised and confounded. O miserable condition of a beggar! If he begs, he is confounded with shame, and if he does not beg, he is consumed with want, and indeed is compelled by necessity to beg. He maintains that God is unjust because he does not dispense properly; he accuses his neighbor of being evil because he does not help fully; he is offended, he complains, he curses. Consider the opinion of the wise man on this subject: "It is better to die than to want." "The poor man shall be hateful even to his own neighbor." "All the days of the poor are evil." "The brethren of the poor man hate him, and even his friends have departed far from him." "When you are happy, you will count many friends. If times are dark, you are alone".»]

21. Ibid.: «Proh pudor! Secundum fortunam estimatur persona, cum pocius secundum personam sit estimanda fortuna. Tam bonus reputatur ut dives, tam malus ut pauper, cum pocius tam dives sit reputandus ut bonus, tam pauper ut malus. Dives, autem, superfluitate resolvitur et iactancia effrenatur, currit ad libitum et corruit in illicitum, et fiunt instrumenta penarum que fuerant oblectamenta culparum. Labor in adquirendo, timor in possidendo, dolor in amittendo mentem eius semper fatigat, sollicitat, et affligit. "Ubi est thesaurus tuus, ibi est et cor tuum”.» 
If thou noon aske, with nede artow so woundid

That verray nede unwrappeth al thy wounde hid!

Maugree thyn heed, thou most for indigence

Or stele, or begge, or borwe thy despence! À voler, mendier, emprunter ton pain.

Thow blamest Crist and seist ful bitterly He mysdeparteth richesse temporal ; Thy neighebor thou wytest synfully, And seist thou hast to lite and he hath al «Parfay», seistow, «somtyme he rekene shal,

Whan that his tayl shal brennen in the gleede,

For he noght helpeth needfulle in hir neede.»

Herkne what is the sentence of the wise: «Bet is to dyen than have indigence; Thy selve neighebor wol thee despise.» If thou be povre, farwel thy reverence! Yet of the wise man take this sentence: "Alle the dayes of povre men been wikke.»

Be war, therfore, er thou come to that prikke!

If thou be povre, thy brother hateth thee, And alle thy freendes fleen from thee, allas!

$\mathrm{O}$ riche marchauntz, ful of wele been yee, $\mathrm{O}$ noble, $\mathrm{O}$ prudent folk, as in this cas ! Youre bagges been nat fild with ambes as, But with sys cynk, that renneth for youre chaunce;

At Cristemasse myrie may ye daunce!

Ye seken lond and see for yowre wynnynges;

As wise folk ye knowen al th'estaat Of regnes; ye been fadres of tidynges And tales, bothe of pees and of debaat. I were right now of tales desolaat, Nere that a marchant, goon is many a yeere,

Me taughte a tale, which that ye shal heere. Ne m'avait appris une histoire - la voici ${ }^{22}$.
Et dénude alors tes blessures cachées.

$\mathrm{Tu}$ as beau faire, l'indigence te force

Tu accuses le Christ en termes amers

De mal répartir les biens ici-bas.

Tu critiques ton prochain injustement, Sous prétexte que tu n'as rien et lui, tout. «Certes», dis-tu, «il devra rendre des comptes

Et alors se brûlera la queue au feu

Faute d'avoir aidé les gens miséreux.»

Écoute les maximes que donne le Sage: Ton prochain lui-même alors te méprise.» Tu es pauvre? adieu le respect qui t'est dû ! Du Sage voici encore une maxime: «Chaque jour du pauvre est un jour sans joie»;

Prends donc garde à ne pas tomber si bas.

Si tu es pauvre, ton frère te haïra

Et tous tes amis, hélas! s'enfuiront.

Ô riches marchands aux biens abondants, Nobles et prudents gens que voici, Vos cornets ne sont pas pleins de doubles as Mais des six et cinq roulent pour vous combler.

À la Noël vous danserez joyeux.

Vous voyagez et surveillez vos gains.

Fort sages vous connaissez l'état Des royaumes. Vous êtes pères de nouvelles Et d'histoires, touchant la paix ou la guerre. Je n'aurais rien ici à raconter Si un marchand, mort il y a longtemps,
Mais si tu t'y refuses, la détresse te navre «Mieux vaut mourir qu'être dans la misère,

22. Geoffrey Chaucer, The Canterbury Tales, «The Man of Law's Prologue», 1. 99-133 (trad A. CRÉPIN). 
Chaucer emploie un intertexte qui a clairement une portée morale et théologique; et il traduit sans grands écarts trois strophes, permettant une identification aisée de l'original très bien connu à l'époque. Puis il crée un dérapage conséquent puisque, loin d'être fustigés pour leurs préoccupations mercantiles, les marchands sont loués d'avoir su devenir riches de biens temporels. Saint Jérôme prônait une traduction de sens à sens plutôt que du mot à $\operatorname{mot}^{23}$. Dans les dernières strophes, nous n'avons ni l'une ni l'autre. Chaucer trahit et le mot et le sens du De miseria d'Innocent III.

Il va loin dans la provocation car les marchands sont dits être nobles et prudents pour cette raison qu'ils savent s'enrichir de biens temporels. Cette prudence est exprimée à travers l'image du jeu de dés, jeu de hasard par excellence : leurs cornets à dés ne sont pas remplis de doubles as (un coup perdant), mais de cinq et six (un coup gagnant). Non seulement les richesses temporelles deviennent les biens désirables, mais elles sont en plus accessibles par de la chance au jeu, soit par l'intervention de Fortuna, allégorie de l'imprévisibilité du sort. La clairvoyance des marchands a partie liée avec Fortune. Pourtant, Prudentia et Fortuna ne sont pas censées faire bon ménage, en particulier dans une traduction qui s'autorise d'un pape.

En outre, la troisième strophe du prologue, celle qui précède la référence aux marchands, reprend deux sentences bibliques (Ecclésiastique 40:28 et Proverbes 14:20). Dans les deux cas, l'auteur de la sentence est désigné comme étant un sage, the wise (113), the wise man (117). Or, les marchands sont qualifiés par le même adjectif au vers 128: «Ye seken lond and see for yowre wynnynges;/ As wise folk ye knowen al th'estaat/Of regnes» (127-129) [«Vous voyagez et surveillez vos gains. Comme vous êtes des gens sages, vous connaissez l'état des royaumes»]. Les marchands sont mis sur un pied d'égalité avec les sages de la Bible par le biais d'une sagesse éminemment temporelle, qui concerne l'état des royaumes, royaumes terrestres et non célestes, et qui a pour résultat l'acquisition de richesses monnayables et en aucun cas spirituelles. Enfin, c'est leur réussite matérielle qui, d'après le Juriste, motivera leur célébration de Noël, soit rien moins que la naissance du Christ.

En ce qui concerne l'emploi de l'adjectif «prudent», Juliette Dor propose l'explication suivante:

Il est peut-être plus osé d'avancer l'idée que voici pour éclaircir le mystère qui entoure «prudent» (PrMLT 124). Ils [les marchands] sont en l'occurrence prudents, puisque... la richesse engendre de grands biens, tandis que la pauvreté

23. T. W. Machan, «Chaucer as Translator», in R. Ellis éd., J. Price, S. Medcalf et P. Meredith collab., The Medieval Translator: The Theory and Practice of Translation in the Middle Ages, Cambridge, 1989, p. 56. 
engendre de grands maux. S'étant assuré la richesse, ces hommes ont fait preuve de prudence ${ }^{24}$.

Cette logique est aisément admissible, mais la contextualisation du concept de prudence ici n'en reste pas moins totalement provocatrice puisqu'elle se greffe sur un discours moral et théologique prônant le mépris des richesses.

Une réaction fréquente vis-à-vis du dérapage opéré dans le prologue du Juriste est d'y voir une manière de caractériser le pèlerin qui prononce ce discours. Les erreurs de celui-ci seraient une manière de camper son personnage. C'est là bien sûr une solution possible. Elle est cependant insatisfaisante. Car le phénomène sémantique du «dérapage contrôlé» apparaît ailleurs chez Chaucer et dépasse un effort de caractérisation ponctuel. Il convient de ne pas réduire les jeux sémantiques du texte à de simples effets comiques visant à ridiculiser un personnage. Loin d'être un aspect isolé, le pervertissement du De miseria d'Innocent III s'accompagne dans l'introduction qui le précède d'un réseau d'autocontradictions et de lapsus intertextuels qui, en définitive, renvoient à la figure et à la position de Chaucer lui-même et à son statut d'auteur. Ainsi, un vecteur expressif majeur vient de la posture auctoriale de Chaucer, dessinée par les provocations textuelles de celui-ci à l'endroit de notre interprétation du corps et de ses émotions.

Dans la dernière strophe du prologue, l'Homme de loi explique à propos des marchands qu'ils sont pères des nouvelles et des contes, fathers of tidings and tales (129-130). Le Juriste tient d'un marchand le conte qu'il s'apprête à raconter, marchand sans lequel il serait maintenant «dépourvu de contes »: of tales desolaat. Le terme desolaat est fort, il est construit sur le latin desolatus «déserté, abandonné», participe passé du verbe desolo «dépeupler, ravager»; il évoque un manque radical. Or, le bien si précieux dont le pèlerin a failli manquer est une histoire à raconter dans un concours dont le but est le divertissement, et le prix un souper gratuit. L'hiatus entre le mot choisi, desolaat, et la raison de la désolation, tout en étant comique, fait écho aux commentaires de l'Homme de loi concernant l'œuvre de Chaucer.

En effet, le Juriste fait référence aux œuvres de Chaucer dans son Introduction. Il explique qu'il est très difficile de trouver un conte idoine, qui n'ait pas déjà été raconté par Chaucer.

But nathelees, certeyn,

I kan right now no thrifty tale seyn
Mais néanmoins, il est vrai,

Je ne connais en ce moment aucun conte adéquat

24. J. Dor, «L'énigme du "Prologue du Conte de l'Homme de loi": Chaucer et l'autoplagiat», dans R. Eluis et R. TiXIER éd., The Medieval Translator. Traduire au Moyen Âge, vol. 5, Turnhout, 1996, p. 385. 
That Chaucer, thogh he kan but lewedly On metres and on rymyng craftily, Hath seyd hem in swich Englissh as he kan Of olde tyme, as knoweth many a man; And if he have noght seyd hem, leve brother,

In o book, he hath seyd hem in another. For he hath toold of loveris up and doun Mo than Ovide made of mencioun In his Episteles, that been ful olde. What sholde I tellen hem, syn they been tolde?
Que Chaucer, malgré ses maladresses En métrique et en rimes habiles,

N'a pas raconté, dans son anglais Du temps jadis, comme beaucoup le savent. S'il n'a pas conté ces histoires, cher frère,

Dans ce livre-ci, c'est dans celui-là. Il a parlé d'amoureux tous azimuts, Plus nombreux que ceux qu'Ovide a cités Dans ses Lettres, qui sont bien anciennes. Pourquoi devrais-je les redire, puisqu'elles ont déjà été racontées ? ${ }^{25}$

Ainsi, le Juriste proclame sa difficulté à trouver un conte que Chaucer n'ait pas déjà raconté, pour expliquer plus tard que son conte lui vient d'un marchand, père des contes, et non de Chaucer.

Il fait ensuite la liste des héroïnes dont Chaucer est censé avoir reproduit les discours dans son livre The Legend of Good Women. Or, le Juriste commet de grosses erreurs, des erreurs évidentes : il donne des noms qui n'apparaissent pas dans l'œuvre de Chaucer, à savoir Déjanire, Pénélope, Hermyon, Léandre, Ladomya et Eleyne, et il omet par contre de nommer deux hérö̈nes dont les légendes s'y trouvent, soit Cléopâtre et Philomena. Cependant, les six noms qu'il inclut abusivement dans la bibliographie de Chaucer appartiennent tous aux Hérö̈des d'Ovide ${ }^{26}$. Chaucer mentionne les Hérö̈des explicitement, en les appelant les Lettres - il s'agit en effet de lettres écrites par des femmes aux hommes qu'elles ont aimés et qui, en général, les ont trahies. Puis, le Juriste dit que Chaucer a été plus prolifique qu'Ovide, mais il attribue à Chaucer des récits qui n'existent que dans les Hérö̈des d'Ovide, produisant le sentiment inverse et confus que Chaucer n'est pas l'auteur de beaucoup d'histoires.

Vient ensuite une remarque véhémente sur une autre hérö̈ne ovidienne, dont la lettre apparaît dans les Héroïdes; je veux parler de Canacé.

But certeinly no word ne writeth he Of thilke wikke ensample of Canacee, That loved hir owene brother synfully Of swiche cursed stories I sey fy! Or ellis of Tyro Apollonius, How that the cursed kyng Antiochus Birafte his doghter of hir maydenhede, That is so horrible a tale for to rede, Whan he hir threw upon the pavement.
Mais c'est un fait qu'il garde le silence Sur le mauvais exemple de Canacé Qui eut pour son frère un amour coupable Horreur de telles histoires maudites ! Ou encore sur Apollonius de Tyr, Comment le maudit roi Antiochus Ravit à sa propre fille son pucelage Horrible, insupportable est cette scène Où on le voit la renverser à terre.

25. Geoffrey Chaucer, The Canterbury Tales, «The Man of Law's Introduction», 1. 45-56 (TdA).

26. Ovide, Hérö̈des, éd. H. Bornecque, trad. M. Prévost, Paris, 1999. 
And therfore he, of ful avysement, Nolde nevere write in none of his sermons Of swiche unkynde abhomynacions, $\mathrm{Ne}$ I wol noon reherce, if that I may.
Aussi, après avoir bien réfléchi,

A-t-il banni de tous ses ouvrages

Ces abominations contre nature.

Et je ferai de même, sauf impossible ${ }^{27}$.

L'Homme de loi réprouve avec force les récits qui portent sur des cas d'inceste, tels précisément les histoires de Canacé et d'Apollonius de Tyr. De ces récits il dit: «Of swiche cursed stories I sey fy!» (80) [ «Je dis fi de telles maudites histoires!»]. Ce passage a généralement été lu comme une plaisanterie faite par Chaucer à l'encontre de son ami John Gower. Car dans le Confessio amantis, Gower raconte et l'histoire de Canacé et celle d'Apollonius de Tyre. Mais là encore, les choses sont complexes. La légende de Canacé et de son frère Macarée concerne une relation incestueuse due à l'ignorance du tabou d'inceste. L'histoire de Canacé est frappante. Chaucer s'arrange pour que son lecteur ait ce récit en tête: il donne la référence des Lettres d'Ovide et il choisit une histoire difficilement oubliable, que Gower reprend en anglais. Puis l'Homme de loi insiste sur le fait que Chaucer jamais n'écrirait sur Canacé: «But certeinly no word ne writeth he/Of thilke wikke ensamble of Canacee» (77-78) [« Mais il est certain que Chaucer n'a jamais écrit un mot de cet exemplum si mauvais, si condamnable de Canacé»]. Or, c'est faux. Car Chaucer est en train d'écrire sur Canacé au moment où son personnage clame que Chaucer jamais n'écrirait sur elle. Mais surtout, Canacé est le personnage principal du «Conte de l'Écuyer» dans ces mêmes Contes de Canterbury qui contiennent le discours du Juriste. Comme nous l'avons vu, «Le Conte de l'Écuyer» est organisé pour que le lecteur s'attende à ce que le récit aboutisse au drame incestueux habituellement associé au nom de Canacé.

Quand un texte comporte, comme c'est le cas ici, des déplacements, des dérapages et des contradictions organisées, le lecteur a deux options interprétatives principales. Il peut considérer qu'il s'agit de bévues, de lapsus involontaires de la part de l'auteur, d'imperfections dues au caractère inachevé de l'œuvre, la tentation interprétative étant alors de rectifier le texte. Une autre option à ce type d'interventionnisme est d'étudier ces déplacements comme autant d'aspects signifiants d'une poétique maîtrisée par l'écrivain. Quand les dérapages sont occasionnels et isolés, la première voie peut à la rigueur se justifier, bien qu'avec prudence. Quand un texte fourmille de contradictions, il me paraît avisé de choisir la deuxième option. Pourtant, il n'est pas rare que la première option soit favorisée par la critique en ce qui concerne «L'Introduction et le Prologue de l'Homme de loi», par exemple quand il s'agit du passage suivant:

27. Geoffrey Chaucer, The Canterbury Tales, «The Man of Law's Prologue», 1. 77-89 (trad. A. CRÉPIN). 
But of my tale how shal I doon this day? Me were looth be likned, doutelees, To Muses that men clepe Pierides-Methamorphosios woot what I mene; But nathelees, I recche noght a bene
Alors comment m'y prendrai-je aujourd'hui? Je ne voudrais pas être comparé Aux Muses appelées les Piérides: Voyez Ovide et ses Métamorphoses. En tous les cas, je me moque comme d'un clou (litt. haricot)

Though I come after hym with hawebake. D'avoir un mauvais rang, après Chaucer. I speke in prose, and lat him rymes make. Je parle en prose et lui laisse les vers ${ }^{28}$.

Le dernier vers de ce passage annonce un conte en prose. L'Homme de loi dit renoncer aux vers devant la maîtrise de Chaucer. Or le conte du Juriste est en vers et en rimes royales. Pour cette raison, il a été régulièrement admis que le conte du Juriste aurait dû être déplacé à l'intérieur des Contes de Canterbury et être attribué à un autre pèlerin ${ }^{29}$. C'est une hypothèse qui ne tient pas compte du réseau textuel global du passage en question, lequel accumule les contradictions.

Aux contradictions déjà observées, il convient d'ajouter la suivante, qui fait lien avec le précédent passage quand il s'agit de l'opposition vers/prose. Dans une strophe ultérieure, l'Homme de loi juge que Chaucer n'est pas très doué pour les rimes et les vers, «thogh he kan but lewedly/On metres and on rymyng craftly » (47-48). Il ajoute également que l'anglais de Chaucer est vieilli, «Hath seyd hem in swich Englissh as he kan/Of olde tyme» (49-50). Chaucer est le nom du narrateur dans Les Contes de Canterbury: le Chaucer de l'Homme de loi est un pèlerin faisant partie de son public, au moment où celui-ci dit que l'anglais de Chaucer est daté. Là encore, cet hiatus a suscité une série de réactions qui visent à rétablir une cohérence interprétative. Il a été proposé que l'Homme de loi n'a pas reconnu Chaucer parmi le groupe de pèlerins et qu'il ne le connaît que de réputation, d'où également sa remarque et ses erreurs bibliographiques concernant The Legend of Good Women. Ou au contraire, certains critiques pensent que l'Homme de loi a reconnu Chaucer mais qu'il entretient des sentiments ambivalents à son égard, raison pour laquelle il attaque son art tout en l'encensant par la suite ${ }^{30}$. Ces interprétations montrent que le texte trouble le confort du lecteur, lequel cherche à ajouter un supplément de fiction à la narration afin de stabiliser et clarifier la position des personnages.

28. Geoffrey Chaucer, The Canterbury Tales, «The Man of Law's Introduction», 1. 90-96 (trad. A. CRÉPIN).

29. L'article le plus récent sur cette question est celui de G. BARLOw, «A Thrifty Tale: Narrative Authority and the Competing Values of the Man of Law's Tale», The Chaucer Review, 44/4 (2010), p. 397-420. Voir aussi A. Blamires, Chaucer, Ethics, and Gender, Oxford, 2006, chap. 4.

30. Cf. W. L. Sullivan, «Chaucer's Man of Law as a Literary Critic», Modern Language Notes, 68/1 (1953), p. 1-8; A. DAvID, «The Man of Law vs. Chaucer: A Case in Poetics», PMLA, 82, 1967, p. $217-225$. 
Il me parait plus utile d'observer le jeu du texte, qui mobilise le lecteur autrement. Je récapitule: l'Homme de loi s'apprête à raconter un conte qu'il tient d'un marchand mort il y a de nombreuses années; il va raconter un conte que Chaucer n'a pas raconté bien que, d'après lui, il soit impossible de trouver un conte que Chaucer n'ait pas déjà narré dans l'un ou l'autre de ses écrits ; l'anglais de Chaucer est dépassé, bien que celui-ci soit présent parmi la troupe de pèlerins qui écoutent le Juriste, à l'inverse du marchand, père de contes qui, lui, est mort depuis longtemps; enfin, Chaucer est mauvais rimeur et versificateur, pourtant l'Homme de loi renonce à dire après lui son conte en rimes, et il dit et ceci et son conte en rimes royales. Enfin, pour clore le tout, Chaucer choisit pour son Juriste le conte de Constance raconté par Gower dans son Confessio amantis, œuvre critiquée par la bande pour ce qu'elle inclut deux récits d'inceste: jamais Chaucer ne se commettrait à parler d'une histoire aussi répréhensible que celle de Canacé. Or, Canacé est l'héroïne de l'un des Contes de Canterbury.

La figure de Chaucer est à la fois établie comme auteur de contes et réfutée comme telle par son propre personnage au moyen d'un déplacement concernant ses œuvres. En outre, Chaucer est en porte-à-faux vis-à-vis du marchand qui a transmis son conte à l'Homme de loi. S'il l'est vis-à-vis de ce marchand, il peut l'être vis-à-vis du groupe que celui-ci représente (les marchands), ce qui le place en opposition avec leur caractéristique première pour l'Homme de loi, à savoir qu'ils sont les pères de récits, fathers of tales. En définitive, la question est posée de savoir ce que signifie être à l'origine d'un discours. La succession de contradictions élaborées par Chaucer pousse à entrevoir une réponse. Chaucer organise un texte qui dit: "Chaucer n'est pas un père de contes, et il n'est pas l'auteur de ce texte: son anglais est daté, le mien ne l'est pas.» Ce faisant, l'originalité de cette affirmation, et surtout l'originalité de sa formulation, crée le sentiment d'une évidence contraire, comme si la voix qui émergeait par-delà la multiplication des discours intradiégétiques de ses pèlerins disait fondamentalement: «Je suis bien à l'origine de ma parole alors même qu'elle est infiltrée de toutes parts.»

Le style kinésique qui ressort de «L'Introduction et Prologue de l'Homme de loi» évoque le timbre d'une voix qui perce à travers les dérapages, et dont les résonances se perçoivent le plus clairement quand cette voix prétend se taire. Le personnage fictionnel Chaucer ne pipe mot quand l'un des pèlerins, né de la plume de l'auteur Chaucer, clame que tous savent combien l'anglais de Chaucer est périmé. L'humour de cette allégation présentifie Chaucer dans le sourire ou le rire qu'il suscite chez le lecteur. Parce que la présence du personnage fictionnel Chaucer est oblitérée de façon paradoxale, le sentiment d'une présence de Chaucer l'auteur est renforcé, comme le texte le fait sentir, par l'hiatus, le pouvoir d'action de cette personne inconnue et définitivement inaccessible, qui est à l'origine du texte que nous avons sous les yeux et auquel nous réagissons. 
Chaucer l'écrivain et Chaucer le narrateur sont deux concepts qui suffisent mal à rendre compte de l'instance qui jongle et feinte sur le seuil du texte. Le travail de harcèlement textuel et de mésinterprétation pratiqué par cette version de l'auteur Chaucer participe d'une tendance à provoquer le lecteur, à le mettre au défi d'un repositionnement interprétatif. De la même manière que cet auteur change de posture et se déplace continuellement, le lecteur, s'il veut le suivre, doit également bouger. Et ce qu'il découvre en définitive n'est pas un sens herméneutique mais son propre mouvement interprétatif. Ainsi, la figure ambivalente de l' "auteur» sert à créer un espace narratif permis par l'acte de lecture, lequel constitue un geste expressif en réponse au geste expressif dont l'œuvre est la trace.

Tim William Machan souligne que Chaucer est de façon fondamentale l'auteur du sens qu'il traduit ${ }^{31}$, mais qu'il était culturellement problématique pour Chaucer d'être un auteur, dans le sens médiéval de auctor, de source d'autorité ${ }^{32}$. Car le statut culturel des langues et littératures vernaculaires au Moyen Âge interdisait aux écrivains vernaculaires d'être appelés «auteurs», en particulier de leur vivant ${ }^{33}$. Par conséquent, Chaucer, en s'érigeant auteur vernaculaire, représentait un paradoxe culturel («by medieval standards Chaucer was a paradox - a "vernacular author" ») ${ }^{34}$. Par sa manière de dévoyer ses sources, Chaucer a donné une voix à une nouvelle version de l'auctor ${ }^{35}$. Ce faisant, il a aussi changé la place du lecteur en le sollicitant d'une manière inédite. Pour opérer ce changement, Chaucer semble avoir compté sur un mode de lecture typiquement médiéval. En effet, la sémiotique médiévale, qui cherche le fruit du sens sous l'écorce de la lettre ${ }^{36}$, produit des lecteurs extrêmement attentifs aux détours du

31. T. W. MACHAN, «Chaucer as Translator», p. 58.

32. Ibid., p. 66. Voir aussi A. J. Minnis, Medieval Theory of Authorship : Scholastic Literary

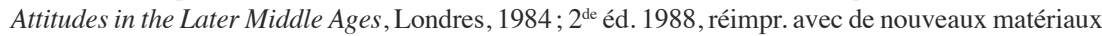
en 2009; et A. J. Minnis, A. B. ScotT et D. Wallace, Medieval Literary Theory and Criticism ca 1100ca 1375: The Commentary-Tradition, Oxford, 1988.

33. T. W. Machan, «Chaucer as Translator», p. 63: «Indeed, throughout most of the Middle Ages the cultural status of vernacular language and literature was such that vernacular writers were precluded from calling themselves "authors".»

34. Ibid., p. 64.

35. R. R. EDWARDS, «Authorship, Imitation, and Refusal in Late-Medieval England», dans G. Bolens et L. ERne éd., Medieval and Early Modern Authorship, Tübingen, 2011, p. 211-233 (SPELL: Swiss Papers in English Language and Literature, 25); H. COOPER, «Choosing Poetic Fathers: The English Problem», ibid., p. 203-221.

36. La métaphore de l'écorce et du fruit est récurrente dans la pensée médiévale, exprimant l'idée d'une vérité orthodoxe à mettre en évidence par l'interprétation. La lettre contient et recouvre la vérité du sens; lire consiste donc à déceler, à découvrir par un travail de déchiffrage symbolique et analogique le sens enfoui par l'auteur, caché dans l'écriture. La distinction du sens et de la lettre apparaît chez Paul dans la deuxième épître aux Corinthiens, 3, 6. Elle est reprise par Augustin dans 
texte puisque c'est par la lettre qu'il est possible d'accéder au sens et à la vérité. Et c'est donc en utilisant la force et les atouts de la sémiologie médiévale que Chaucer a subverti le système interprétatif médiéval. Le style kinésique de la poétique chaucérienne opère à travers la mobilité imprévisible d'un auteur liminaire et la dynamique des destinataires aléatoires de ses gestes expressifs. L'espace narratif qui donne lieu à cette interaction n'est ni la mémoire accumulative et désincarnée d'un lecteur idéal, ni la légitimité de l'auctor traditionnel, garant non moins idéalisé d'un sens à découvrir, mais bien l'espace d'un échange interpersonnel à la fois précis et fortuit - espace actualisé par la rencontre du discours chaucérien et de la réactivité épistémologique, émotionnelle et corporelle d'un destinataire au profil historique et personnel nécessairement variable.

Guillemette BoLENS - Université de Genève, Faculté des Lettres, Département de langue et littérature anglaises, Uni Bastions 5, rue de Candolle, 1211 Genève $4, \mathrm{CH}$

\section{La narration des émotions et la réactivité du destinataire dans Les Contes de Canterbury de Geoffrey Chaucer}

Dans Les Contes de Canterbury, Chaucer met en évidence les rapports de force complexes qui s'actualisent à travers l'expression verbale et corporelle des émotions. Ces rapports de force sont manifestes entre les personnages des Contes de Canterbury, mais ils impliquent également les destinataires du texte. L'écrivain Chaucer, quand il met en récit l'expression corporelle d'une émotion, lance un défi interprétatif à ses destinataires. Mon objectif est ainsi de chercher à comprendre ce que le texte signale de nos modalités d'accès à un sens émotionnel permis par une narration des signes corporels.

Chaucer-Les Contes de Canterbury - «Le Conte de l'Écuyer »- «L'Introduction et le Prologue de l'Homme de loi» - Canacé - amour courtois - style kinésique.

son De doctrina Christiana (3.7.11). Et elle est explicitée par l'allégorie de Raison dans Le Roman de la Rose de Jean de Meung, lors du discours de Raison à l'Amant (Guillaume De Lorris et JEAN De Meung, Le Roman de la Rose, éd. et trad. d'A. Strubel, Paris, 1992, 1. 7164-7170). Une bonne lecture de l'écorce, du sens littéral, offre une voie d'accès au sens symbolique et à la vérité du sens spirituel contenu, comme un fruit, dans l'écriture. La métaphore du fruit et de l'écorce, qui sert à signifier la distinction de la lettre et du sens, a été employée par Bède le Vénérable, Raban Maur et Alain de Lille (Bède, PL 91, col. 808; Raban Maur, PL 109, col. 192; Alain de Lille, De planctu naturae, PL 210, col. 451). Elle est également utilisée par Chaucer dans Les Contes de Canterbury à la fin du «Conte de l'Aumônier» («The Nun's Priest's Tale», 1. 3438-3443), qui est une fable sur un coq, une poule et un renard. 


\section{The Narration of Emotions and the Reader's Reactions in Geoffrey Chaucer's Canterbury Tales}

In The Canterbury Tales, Chaucer highlights the complex power relationships that are enacted through the verbal and corporeal expression of emotions. These power relationships are manifest between all protagonists of the narrative, but they also involve the readers. When Chaucer narrates the corporeal expression of an emotion, he wittingly challenges the interpretive acumen of his readers. My purpose in this essay is to analyze the way in which Chaucer calls attention to our modes of access to emotional meanings elicited by the narration of corporeal signs.

Chaucer - The Canterbury Tales - «The Squire's Tale» - «The Man of Law's Introduction and Prologue»-Canacee - courtly love - kinesic style. 
